\title{
Research methods of metabonomics and its applications in acupuncture
}

\author{
Jin Junmei MSc, Xuan Lihua MSc
}

J Junmei, X Lihua. Research methods of metabonomics and its applications in acupuncture. Curr Res Integr Med 2015;1(2):26-28.

Metabonomics is a new 'omic' that has emerged in the postgenomic era. Recently, metabonomics technology is increasingly and widely used in the field of traditional Chinese medicine research; however, metabonomics research investigating the function and mechanisms of acupuncture is still at an exploratory stage. The present article describes the unique

$\mathrm{M}$ etabonomics is a new 'omic' that has emerged in the postgenomic era. It has the advantages of high throughput, sensitivity and accuracy. To date, the application of metabonomics technology in research has been preliminary, and there is considerable potential for its application in research investigating traditional Chinese medicine. Both acupuncture and metabonomics technology are superior techniques, but metabonomics research related to the function and mechanism of acupuncture is still in the exploratory stage, and there has been relatively little research in this field. Applying metabonomics to studying acupuncture will help to elucidate the mechanism of acupuncture thoroughly and systematically, and will promote acupuncture theory and clinical development. The present article describes both the definition and research methods of metabonomics, as well as its potential application in acupuncture.

\section{DEFINITION OF METABONOMICS}

Nicholson et al (1) first proposed the definition of metabonomics in 1999. 'Metabonomics' refers to the quantitative analysis of all metabolites in a biological system. Fiehn (2), Nielsen and Oliver (3), and other authors have proposed different but related definitions of metabonomics, which include: metabolic profiling analysis (quantitative determination of specific predetermined structure- and property-related metabolites); metabolite target analysis (quantitative determination of products or substrates of a target protein); and metabolite fingerprinting (qualitatively and semiquantitatively analyzing internal and external metabolites of cells).

Metabonomics research is based on the analysis of metabolic group indexes and uses high-throughput tests and different data processing techniques and targets to model biological information, which is collected and systematically meta-analyzed. It represents an important branch of systems biology, and is another research field and branch of systems biology after genomics, proteomics and transcriptomics. It is a research method that quantitatively analyzes a variety of time-related responses of metabolic parameters when an organism is affected by exogenous stimuli or genetic modification (4). Metabonomics studies the change of all small molecule metabolites with molecular weight $<1000 \mathrm{Da}$ when the organism is subject to exogenous stimuli. As a new methodology, metabonomics has increasingly become one of the cutting-edge methods of studying health and disease worldwide. All exogenous stimuli, such as drugs, foods, acupuncture and other factors, will lead to changes in the metabolites in an organism. The changes in advantages of metabonomics in systems biology, analyzes the application of metabonomics, and presents a preliminary exploration of the application of metabonomics in modern acupuncture research including sham acupuncture, time acupuncture, mechanistic research investigating the effects of acupuncture on serious illness and the compatibility of prescriptions with acupuncture in clinical research.

Key Words: Acupuncture; Metabonomics

the genome and proteome will be amplified at the metabonomics level and can, thus, be easily and conveniently tested; in addition, the number of metabolites to be assessed is lower than the number of genes and proteins that can be assessed in the genome and proteome. Thus, metabonomic research methods have considerable theoretical significance in the diagnosis of many types of diseases, drug effects, safety evaluation and mechanistic research $(5,6)$.

\section{METABONOMICS RESEARCH METHODS}

Metabonomics research methods include nuclear magnetic resonance (NMR), mass spectrometry (MS), high-performance liquid chromatography (HPLC) and other modern analytical techniques. These combine metabolic information and knowledge of pathophysiological processes by determining and analyzing the change in metabolites in cells, tissues and humoral extracts in organisms over time to detemine the metabolic processes of an organism $(7,8)$. The analysis process mentioned above includes sample preparation, data acquisition, analysis and interpretation $(9,10)$, which reflects its advantages of high throughput, sensitivity and accuracy. As for metabonomics research, a spectra of biological samples are first produced by chemical processing. NMR is an efficient and noninvasive detection method among the currently available methods, and is universally applicable to hydrogenbearing metabolites; HPLC has high resolution and throughput; and MS has the characteristics of universality, high sensitivity and specificity. These methods are the main tools for analysis used at present. Combining those methods, which have high sensitivity and better repeatability, can lead to the detection of every kind of metabolite. NMR alone can detect data on hundreds of metabolite components in biological samples, and does not require the properities of materials to be detected in advance. Meanwhile, NMR requires less sample, does not require complicated sample preparation or derivatives, and the sample can be recovered; thus, NMR is currently one of the most common research methods used in metabonomics (11). Given each sample's original data matrix, standardization and other preprocessing methods, all detected data can be summed to enable metabonomics data to be used in multivariate statistic analysis (12). Finally, according to the target to be analyzed and practical problems to be solved, a statistical model is chosen to conduct model recognition analysis, the model is assessed by data dimensionality reduction and unsupervised analysis is conducted. Selecting one type of sample tested to conduct mathematics modelling, perform variable weighting processing, ensure

Hangzhou Economic and Technological Development Zone, Zhejiang Hospital of Traditional Chinese Medicine, Hangzhou, China

Correspondence: Xuan Lihua, Zhejiang Hospital of Traditional Chinese Medicine, 54 Post Road, Hangzhou, Zhejiang, China, 310006.

Telephone 86 13588420630, fax 86-057186919382, e-mail xlh1083@163.com 
the number of main components to be analyzed and, finally, use supervised statistics to distinguish unknown samples. Principal component analysis is the most common unsupervised model recognition method in metabonomics analysis and also the most common model recognition method that analyzes and screens characteristic metabolites according to the results of multivariate statistical analysis $(13,14)$.

\section{THE APPLICATION OF ACUPUNCTURE RESEARCH IN METABONOMICS}

The characteristic of metabonomics is that it can quantifiably represent the sum of endogenous metabolites in an organism and the response of that organism to internal and external changes. The metabolism of the body is in dynamic equilibrium, but when an organism is influenced by varying pathological and physiological factors, it will generate metabolic changes in response at the cell, tissue and whole-body levels, and the concentration of metabolites will change. Metabonomics detects overall and dynamic changes in metabolites, and extracts and screens related markers of biological metabolism. On this basis, metabonomics identifies the metabolic pathways or links that are influenced, analyzes corresponding changes in the proteome, ensures regulation mechanism of the metabolic network and then links with and determines related functions of regulatory genes. Metabonomics has been widely used in various studies investigating the mechanisms of drugs $(15,16)$, but has not been widely used in studies investigating acupuncture for the treatment of various diseases.

Most clinical studies investigating acupuncture-moxibustion are merely imitating Western clinical research standards, as with applying metabonomics to investigating sham acupuncture. In addition to the lack of rigorous randomized controlled trials involving large samples, there are considerable problems in acupuncture research both in China and abroad, including the application of blinded methods, appropriate allocation concealment, accurate description of depigmentation rate, long-term effect and cost estimation. Of these, how to obtain ideal sham acupuncture is the most significant challenge for clinical acupuncture-moxibustion research $(17,18)$. This is because an appropriate combination between acupuncture research and modern clinical research has not been found. The true therapeutic effect of acupuncture should be based on the theory of Chinese medicine acupuncture, four methods of diagnosis, accurate differentiation of syndromes and treatment, and acupuncture-moxibustion techniques. The results of foreign clinical trials investigating acupuncture (which lack an appropriate combination of traditional theory and modern experimental design of clinical research) and acupuncture treatment conducted by traditional Chinese medicine acupuncturists (who lack knowledge of research techniques) have always generated controversy.

Metabonomics enables unbiased analysis of the living body and dynamic testing of the changes in most small-molecule metabolites of the body. Metabonomics generates authentic and objective results, provides an objective index for exploring the true effect of acupuncture, can distinguish between genuine acupuncture and sham acupuncture and is appropriate for observing long-term effects of acupuncture.

Metabonomics can also be applied to the investigation of time acupuncture. Time acupuncture, which specifically reflects the 'correspondence between man and universe' theory of traditional Chinese medicine, is a characteristic component of acupuncture theory and the clinical application of acupuncture. Time acupuncture techniques include midnight-noon ebb flow, eight methods of intelligent turtle, and the method of eight flight. It also involves computation and deduction on the basis of Zhouyi theory and a set of complex, detailed power calculations to determine treatment time and acupoints to be treated. Due to its characteristic of combining space with time to perform acupuncture and moxibustion therapy, there are difficulties to conducting experiment research involving time acupuncture. At present, most studies investigating time acupuncture are simple observations of the clinical effect and individual indicators. One of the main problems is the lack of reasonable, rigorous experimental design applied to time acupuncture research; another is that there are few reports describing studies that select several appropriate indexes to comprehensively examine the mechanism of acupuncture $(19,20)$. The dynamic nature of metabonomics can make up for this disadvantage, and the various means of collecting urine, saliva and sputum for testing make sampling easy and convenient to continuously and dynamically observe the change in small molecule metabolites that may be produced, which can combine space with time for these investigations. Thus, the application of metabonomics to time acupuncture may facilitate considerable progress for research investigating the mechanism of acupuncture.

Metabonomics as an advanced technology of systems biology has made considerable contributions to early diagonosis of serious illness, toxicology and pharmacology. Early, accurate diagnosis of tumours, such as liver cancer and ovarian cancer, is a distinguishing feature and advantage of metabonomics. Valine, saturated lipids, glycine, lactate, inositol, nucleotides, polyunsaturated fatty acids, taurine and other tumour-related metabolic markers can be identified by metabonomics, which makes metabonomics increasingly applicable to diagnosis, therapy and prognostic evaluation (21). Acupuncture has a therapeutic effect for tumours: it can improve symptoms, prolong the lifespan of patients, improve the quality of life, relieve cancer pain syndrome and, especially, improve the side effects of radiotherapy and chemotherapy, eg, myelosuppression, gastrointestinal reaction, fever etc. Acupuncture treatment of tumour possesses many features and advantages (22). Applying metabonomics to research investigating acupuncture treatment of tumours both gives play to the sensitive detection advantage of metabonomics for tumour diagnosis, treatment assessment and exploring the mechanism of acupuncture treatment at deeper, more extensive and more dynamic levels. In addition, there are some reports investigating Alzheimer diseases research at home and abroad $(23,24)$, but few in application research of venereal disease.

Applying metabonomics to conduct research investigating the prescription compatibility of acupuncture and applying meridian and acupoint to research, researchers explain the phenomenon of meridian and acupoint from various angles and aspects, but no one can clearly explain the research of meridian and acupoints. Research investigating the prescription compatibility of acupuncture on this basis mainly relates to the following points. The acupuncture prescription selected mainly involves simple and specific acupoints, such as shu-mu points, yuan-source points and collateral-points combination, which is not consistent with tradition and reality (25). Also, the detection method of acupuncture and moxibustion prescription studies is generally based on measurement of a single index and rarely involves multilevel, multitarget and multinetwork research, which is general in acupuncture research (26).

An important trait of the application of metabonomics to traditional Chinese medicine research is that it turns measuring a single biomarker to measuring a group of specific metabolic markers, which can reflect all metabolite information of a test sample whose molecular weight is $<1000 \mathrm{Da}$. Thus, it possesses the traits of high throughput and high sensitivity; coordinating acupuncture research of multiindexes and multilevel, it could bring new hope and progress to acupuncture research in future studies.

FOUNDATION SUPPORT: The first batch of "Pine" personnel training project of Zhejiang Hospital of Traditional Chinese Medicine (2001437).

\section{REFERENCES}

1. Nicholson JK, Lindon JC, Holmes E. Metabonomics: Understanding the metabolic responses of living systems to pathophysiological stimuli via multivariate statistical analysis of biological NMR spectroscopic data. Xenobiotica 1999;29:1181-9. 
2. Fiehn O. Metabolic networks of Cucurbitamaxima phloem. Phytochemistry 2003;62:875-86.

3. Nielsen J, Oliver S. The next wave in metabolome analysis. Trends Biotechnol 2005;23:544-6.

4. Rawi Ramautar1, Ruud Berger1,Jan van der Greef, et al. Human metabolomics: Strategies to understand biology. Curr Opin Chem Biol 2013;17:841-6.

5. Dunn WB, Broadhurst DI, Atherton Hi, Goodacre R, Griffin JL. Systems level studies of mammalian metabolomes: The roles of mass spectrometry and nuclear magnetic resonance spectroscopy. Chem Soc Rev 2011;40:387-426.

6. Patti GJ, Yanes O, Siuzdak G. Innovation: Metabolomics: The apogee of the omics trilogy. Nat Rev Mol Cell Biol 2012;13:263-9.

7. Mamas M, Dunn WB, Neyses L, Goodacre R. The role of metabolites and metabolomics in clinically applicable biomarkers of disease. Arch Toxicol 2011;85:5-17.

8. G. Poste. Bring on the biomarkers. Nature 2011;469:156-7.

9. Clayton TA, Baker D, Lindon JC, Nicholson JK. Pharmacometabonomic identification of a significant host-microbiome metabolic interaction affecting human drug metabolism. Proc Natl Acad Sci U S A 2009;106:14728-33.

10. Chen R, Mias GI, Li-Pook-Than J, et al. Personal omics profiling reveals dynamic molecular and medical phenotypes. Cell 2012;148:1293-307.

11. Ramautar R, Somsen GW, de Jong GJ. CE-MS for metabolomics: Developments and applications in the period 2010-2012. Electrophoresis 2010;32:86-98

12. Wishart DS, Jewison T, Guo AC, et al. HMDB 3.0 - the Human Metabolome Database in 2013. Nucleic Acids Res 2012;41:D801-D807.

13. Theodoridis GA, Gika HG, Want EJ, Wilson ID. Liquid chromatography-mass spectrometry based global metabolite profiling a review. Anal Chim Acta 2012;71:7-16.

14. Visanwanathan A, Godin O. Impact of MRI markers in subcortical vascular dementia: A multi-modal analysis in CADASIL. Neurobiology of Aging 2008;31:1629-36.
15. Salek RM1, Xia J, Innes A, et al. AmyInnes A metabolomic study of the CRND8 transgenic mouse model of Alzheimer's disease. Neurochem Int 2010;56:937-47.

16. Keshari KR, Kurhanewicz J, Jeffries RE, Wilson DM, Dewar BJ, VanCriekinge M. Hyperpolarized (13)C spectroscopy and an NMRcompatible bioreactor system for the investigation of real-time cellular metabolism. Magn Reson Med 2010;63:322-9.

17. Liang FR, Ren YL, Tang Y. Current situations about studies on the evaluation of clinical efficacy of acupuncture. Zhen Ci Yan Jiu 2008;33:62-4.

18. Liu J, Wang J-Y, J-L Liu, et al. Placebo control in the design of acupuncture clinical trials. Acupuncture Reasearch 2007;32:389-92.

19. Liang W-Y. Experimental research and clinical application profile of time acupuncture. Chinese Med Res 2006;19:54-6.

20. Zhang Y, Zhang Y, Xing Y-R. Research review of midnight-noon ebb-flow acupuncture in recent ten years. Journal of Shanxi College of Traditional Chinese Medicine 2005;28:60-1.

21. Ma Y-L, Qin H-L. Metabonomics and its Application in oncobiology research. World Chinese Journal of Digestology 2008;16:3877-83.

22. Lv-Lin. Clinical and experimental research of acupuncture's adjustment to tumor immune. Liaoning Journal of Traditional Chinese Medicine 2001;28:638-40.

23. Wu Q-F, Guo L-L, Yu S-G. A 1HNMR-based metabonomic study on the SAMP8 and SAMR1 mice and the effect of electroacupuncture. Exp Gerontol 2011;46:787-93.

24. Mishur RJ, Rea SL. Applications of mass spectrometry to metabolomics and metabonomics: Detection of biomarkers of aging and of age-related diseases. Mass Spectrom Rev 2011;31:70-95.

25. Huang $\mathrm{T}$. The rules of acupuncture points during different periods. Journal of Clinical Acupuncture and Moxibustion 2004;20:1-3.

26. Gao J, Liu X-G, Yan X-Z, et al. Primary analysis on the methodology and strategies for studying mechanisms of acu-moxibusttion by using metabonomics. Acupuncture Res 2011;36:296-301. 